\title{
ASYMPTOTIC BOUNDARY CONDITIONS FOR DISSIPATIVE WAVES: GENERAL THEORY
}

\author{
THOMAS HAGSTROM
}

\begin{abstract}
An outstanding issue in the computational analysis of time-dependent problems is the imposition of appropriate radiation boundary conditions at artificial boundaries. In this work we develop accurate conditions based on the asymptotic analysis of wave propagation over long ranges. Employing the method of steepest descent, we identify dominant wave groups and consider simple approximations to the dispersion relation in order to derive local boundary operators. The existence of a small number of dominant wave groups may be expected for systems with dissipation. Estimates of the error as a function of domain size are derived under general hypotheses, leading to convergence results. Some practical aspects of the numerical construction of the asymptotic boundary operators are also discussed.
\end{abstract}

\section{INTRODUCTION}

Many interesting and important problems involving wave propagation in dissipative systems are posed on unbounded spatial domains. Examples from viscous or turbulent fluid dynamics include the modeling of internal flows, which leads to cylindrical domains, and the modeling of flows past bodies in the ocean or atmosphere, which may be posed on exterior domains. In these cases, even for high Reynolds numbers, viscous regions such as wakes and boundary layers may extend to the far field. Other examples are provided by reaction-diffusion equations, which have been used in models of combustion and population dynamics.

For purposes of numerical computation, an artificial boundary is often introduced. For long-time computations the interaction of the solution and the artificial boundary cannot be avoided. Striking instances of this occur in fluid dynamics when vortices or eddies reach the boundary, though more subtle effects can also be important. These interactions may result in unacceptably large errors throughout the computational domain, especially if the system has instabilities. (For an example of this in a reaction-diffusion system see Hagstrom and Keller [11].)

Received December 12, 1989.

1980 Mathematics Subject Classification (1985 Revision). Primary 65M99, 35B40.

Supported, in part, by the Institute for Computational Mechanics in Propulsion (ICOMP), NASA, Lewis Research Center, Cleveland, Ohio, and by NSF Grant No. DMS-8905314. 
The primary purpose of this work is to develop accurate boundary conditions to be imposed at such boundaries. We are also interested in establishing error estimates and convergence theorems, standard components of theoretical numerical analysis which seem rarely to have been studied in the context of time-dependent partial differential equations on unbounded domains. For linear, separable differential operators, the exact boundary conditions may be represented in terms of appropriate eigenfunction expansions and transform variables. (See, e.g., Gustafsson and Kreiss [7].) For example, suppose $x$ is the spatial coordinate normal to the boundary and the $x$-dependence of the transform solutions takes the form $e^{\lambda_{l}(s) x}$ with $s$ the dual variable to time and $l$ indexing a tangential normal mode. An exact relation at the boundary is given in transform space by

$$
\left(\frac{\partial}{\partial x}-\lambda_{l}(s)\right) \hat{u}_{l}=0 .
$$

Unfortunately, the expression of this relation in the original variables is typically nonlocal in both space and time. For computational efficiency, the added storage and arithmetic operations required by the implementation of nonlocal conditions must, to the extent possible, be avoided. Ideally, local boundary operators would be used. These may be obtained using polynomial or rational approximations to the dispersion relation, $\lambda_{l}(s)$, which in turn can generally be accurate only in a restricted neighborhood of transform space.

An approach to the derivation of boundary conditions is, evidently, to identify region(s) in transform space where polynomial or rational approximations are to be made and, then, to compute the coefficients of the approximation. In their pioneering study of hyperbolic problems, Engquist and Majda [5] considered a particular high-frequency limit. For problems with dissipation, on the other hand, this limit is less likely to lead to accurate results. In this work we consider the use of asymptotic expansions of waves propagating over long distances computed using the method of steepest descent. A consequence of the dissipative terms in the equations studied here is the association of growth or decay with each wave group. By locating minimum decay (maximum growth) rates, we locate appropriate regions for the required approximations. That is, we identify a small number of dominant wave groups, characterized by $\left(\tilde{s}_{i}, l_{i}\right)$, and compute local linear approximations to the dispersion relation,

$$
\lambda_{l_{i}}(s) \approx \lambda_{l_{i}}\left(\tilde{s}_{i}\right)+\lambda_{l_{i}}^{\prime}\left(\tilde{s}_{i}\right)\left(s-\tilde{s}_{i}\right) .
$$

Substituting this into (1) leads to a local operator. The complete asymptotic boundary condition is defined by the composition of a small number of these operators:

$$
\prod_{i}\left(\frac{\partial}{\partial x}-\lambda_{l_{i}}\left(\tilde{s}_{i}\right)-\lambda_{l_{i}}^{\prime}\left(\tilde{s}_{i}\right)\left(\frac{\partial}{\partial t}-\tilde{s}_{i}\right)\right) u=0 .
$$

In $\S \S 2$ and 3, we present in detail the asymptotic analysis and the subsequent derivation of asymptotic boundary conditions. Some numerical considerations 
are discussed in $\S 4$. In $\S 5$ we derive estimates of the error as a function of the size of the computational domain. These lead to the convergence of the solution of the problem on the truncated domain to the solution on the full domain.

A primary motivation of this work is the development of accurate boundary conditions at artificial boundaries for the Navier-Stokes equations. This is carried out in [10] for incompressible flows, where extensive numerical experiments are described. Earlier applications of some of the ideas given here appear in $[8,9]$. Boundary conditions for similar equations with constant coefficients have been derived by Halpern [12] and Halpern and Schatzman [13].

\section{ASYMPTOTIC EXPANSIONS}

We consider, for definiteness, a general system of equations in a semiinfinite channel:

$$
\begin{gathered}
\frac{\partial u}{\partial t}+U \frac{\partial u}{\partial x}+V \frac{\partial u}{\partial y}+W u=A \frac{\partial^{2} u}{\partial x^{2}}+B \frac{\partial^{2} u}{\partial y^{2}}, \\
x \geq 0, \quad y_{0} \leq y \leq y_{1} .
\end{gathered}
$$

These are supplemented by boundary and initial conditions defining a signalling problem:

$$
\begin{gathered}
u(x, y, 0)=0, \\
D_{0} u\left(x, y_{0}, t\right)=0, \\
D_{1} u\left(x, y_{1}, t\right)=0, \\
E_{0} u(0, y, t)=g(y, t) .
\end{gathered}
$$

We assume that the matrices $D_{0}, D_{1}$, and $E_{0}$ are such that the problem is well-posed. We allow a stratified medium; that is, $U, V, W, A$, and $B$ are functions of $y$. Note that equation (4) may be a far-field approximation to a problem whose coefficients are either nonlinear or functions of $x$.

A representation of the solution of problem (4)-(9) may be obtained by means of Laplace transforms and eigenfunction expansions. The eigenvalue problem to be solved is:

$$
\begin{gathered}
s v_{l}+\lambda_{l} U v_{l}+V \frac{d v_{l}}{d y}+W v_{l}=\lambda_{l}^{2} A v_{l}+B \frac{d^{2} v_{l}}{d y^{2}}, \quad y_{0} \leq y \leq y_{1}, \\
D_{0} v_{l}\left(y_{0} ; s\right)=0, \quad D_{1} v_{l}\left(y_{1} ; s\right)=0 .
\end{gathered}
$$

For solutions, $u$, which grow at most exponentially in time we may restrict attention to eigenvalues, $\lambda_{l}$, satisfying

$$
\mathfrak{R}\left(\lambda_{l}(s)\right)<0, \quad \mathfrak{R}(s) \text { sufficiently large. }
$$

We denote by $\mathcal{N}$ the set of indices of eigenvalues which meet the condition above and will refer to the function $\lambda_{l}(s)$ as the dispersion relation. Let

$$
\hat{g}(y ; s)=\int_{0}^{\infty} e^{-s t} g(y, t) d t .
$$


The assumption of well-posedness then implies the existence of a unique collection of functions $\hat{c}_{l}(s)$ such that

$$
E_{0}\left(\sum_{l \in \mathcal{N}} \hat{c}_{l}(s) v_{l}(y ; s)\right)=\hat{g}(y ; s) .
$$

If $c_{l}(t)$ is the inverse transform of $\hat{c}_{l}(s)$, a final expression for $u$ may be obtained:

$$
\begin{gathered}
u(x, y, t)=\sum_{l \in \mathcal{N}} u_{l}(x, y, t), \\
u_{l}(x, y, t)=\int_{0}^{t} c_{l}(p) q_{l}(x, y, t-p) d p,
\end{gathered}
$$

where

$$
q_{l}(x, y, t)=\frac{1}{2 \pi i} \int_{C} e^{s t+\lambda_{l}(s) x} v_{l}(y ; s) d s
$$

and $C$ is an appropriate inversion contour.

To compute asymptotic expansions of $u$, valid for $x$ large, we must evidently find expansions of $q_{l}$. If (17) is evaluated along rays $t=\gamma x, x \gg 1$, the exponent becomes

$$
x\left(\gamma s+\lambda_{l}(s)\right) \text {. }
$$

In order to use the method of steepest descent, we seek points $s^{*}$ such that

$$
\begin{gathered}
\lambda_{l}^{\prime}\left(s^{*}\right)=-\gamma, \\
\mathfrak{R}(\gamma)>0, \\
\mathfrak{I}(\gamma)=0 .
\end{gathered}
$$

Then, assuming that for $0 \leq \gamma_{\min } \leq \gamma \leq \gamma_{\max } \leq \infty$ there exists $s^{*}(\gamma)$ satisfying (19)-(21) with inversion contour, $C$, which can be deformed to the steepest descent path, we have:

$$
\begin{gathered}
q_{l}(x, y, t) \sim e^{x\left(s^{*}(t / x)(t / x)+\lambda_{l}\left(s^{*}(t / x)\right)\right)} \frac{v_{l}\left(y ; s^{*}(t / x)\right)}{\sqrt{2 \pi \lambda_{l}^{\prime \prime}\left(s^{*}(t / x)\right) x}} \equiv \phi_{l}(x, y, t), \\
\gamma_{\min } \leq t / x \leq \gamma_{\max } .
\end{gathered}
$$

Substituting these into (16) formally yields an approximation of $u_{l}$ for $t>$ $\gamma_{\min } x$ :

$$
u_{l}(x, y, t) \sim \int_{\max \left(0, t-\gamma_{\max } x\right)}^{t-\gamma_{\min } x} c_{l}(p) \phi_{l}(x, y, t-p) d p .
$$

This representation has a simple interpretation: the signal data, $c_{l}$, generates wave packets which propagate at their group velocity. At the point $(x, t)$, $x \gg 1$, the solution is approximately the superposition of waves generated at times varying from $t-\gamma_{\max } x$ for the slowest waves to $t-\gamma_{\min } x$ for the fastest. 
Now consider the specialization of these results to hyperbolic systems. In particular, we take the wave equation written as a first-order system:

$$
A=B=W=O, \quad U=\left(\begin{array}{ll}
0 & 1 \\
1 & 0
\end{array}\right), \quad V=\left(\begin{array}{cc}
1 & 0 \\
0 & -1
\end{array}\right),
$$

and suppose that $y_{0}=0$ and $y_{1}=1$. For an appropriate choice of boundary conditions we have

$$
\begin{aligned}
& \lambda_{l}(s)=-\sqrt{s^{2}+l^{2} \pi^{2}}, \\
& \frac{d \lambda_{l}}{d s}=\frac{-s}{\sqrt{s^{2}+l^{2} \pi^{2}}} .
\end{aligned}
$$

From (27) we see that group velocities ranging from 0 to 1 are associated with values of $s=i \omega,|\omega| \geq l \pi$. Furthermore, $\mathfrak{R}(\lambda)=0$; that is, the wave packets do not decay exponentially as they propagate. These observations hold in general for the high frequencies of all hyperbolic problems. (See, e.g., [15].)

For problems with dissipation, on the other hand, it may be possible to further simplify the results. Then, some exponential decay rate may be associated with each wave group. That is,

$$
\mathfrak{R}\left(\gamma s^{*}+\lambda_{l}\left(s^{*}\right)\right) \neq 0 .
$$

For general signal data the large $x$ behavior will be dominated by the wave group with least decay (which may be growth for problems with instabilities). Therefore, we seek $\gamma$ such that the expression above is maximized. Setting to zero the derivative of the decay rate with respect to $\gamma$ yields

$$
\mathfrak{R}\left(s^{*}+\frac{d s^{*}}{d \gamma}\left(\gamma+\lambda_{l}^{\prime}\right)\right)=0,
$$

which by (19) reduces to

$$
\mathfrak{R}\left(s^{*}(\gamma)\right)=0 .
$$

That is, assuming (19) defines a curve in $s$ space, critical points of the decay rate occur as the curve crosses the imaginary axis.

Suppose, for simplicity, that a unique solution of $(30), \tilde{\gamma}_{l}$, exists. (If $s^{*}$ is imaginary, its complex conjugate must also be used.) So long as this wave packet is excited by the initial data, we expect that the dominant contribution to the convolution integral defining $u_{l}(x, y, t)$ will come from a neighborhood of

$$
(t-p) / x=\tilde{\gamma}_{l} .
$$

Introducing a local approximation to $\phi_{l}$ we obtain

$$
\begin{gathered}
u_{l}(x, y, t) \sim \int_{\min \left(0, t-\gamma_{\max } x\right)}^{t-\gamma_{\min } x} c_{l}(p) e^{\lambda_{l}^{0} x} F\left(x+\frac{t-p}{\lambda_{l}^{1}}, t-p\right) v_{l}^{0}(y) d p \\
F(z, \tau)=e^{s^{*} z \lambda_{l}^{1}+\left(\lambda_{l}^{1}\right)^{3} z^{2} / 4 \lambda_{l}^{2} \tau} \frac{1}{\sqrt{-4 \pi \lambda_{l}^{2} \tau / \lambda_{l}^{1}}} .
\end{gathered}
$$


Here we have

$$
\begin{gathered}
\lambda_{l}^{0}=\tilde{\gamma}_{l} s^{*}+\lambda_{l}\left(s^{*}\right), \\
\lambda_{l}^{1}=\frac{d \lambda_{l}}{d s}\left(s^{*}\right)=-\tilde{\gamma}_{l}, \\
\lambda_{l}^{2}=\frac{1}{2} \frac{d^{2} \lambda_{l}}{d s^{2}}\left(s^{*}\right),
\end{gathered}
$$

where $s^{*}$ is evaluated at $\tilde{\gamma}_{l}$. Also we assume that $t>\tilde{\gamma}_{l} x$. The expressions above represent a restriction to the neighborhood of a single point in the dispersion relation. In what follows this restriction will enable us to find an asymptotic boundary condition which consists of local operators. Furthermore, techniques will be given for the numerical computation of the various quantities defined in (34)-(36).

\section{CONSTRUCTION OF THE BOUNDARY CONDITIONS}

We now suppose that an artificial boundary is located at $x=\tau$. The representation of the solution (15)-(16) may be manipulated to yield a variety of exact relationships at the boundary. For example, if the matrix $A$ is positive definite, a characterization of the exact boundary conditions is that a collection of functions, $r_{l}(t)$, exists such that

$$
\left(\begin{array}{c}
\frac{\partial u}{\partial x}(\tau, y, t) \\
u(\tau, y, t)
\end{array}\right)=\sum_{l \in \mathcal{N}} \int_{0}^{t} r_{l}(p)\left(\begin{array}{c}
\frac{\partial q_{l}}{\partial x}(\tau, y, t-p) \\
q_{l}(\tau, y, t-p)
\end{array}\right) d p
$$

In many cases the unknown functions $r_{l}$ may be eliminated to yield a direct relationship between $u$ and $\frac{\partial u}{\partial x}$. Of course, this condition will be nonlocal in $y$ and $t$ and, in general, too difficult to use.

If, however, the asymptotic expansion given in (32) is valid, it may be used to develop a local asymptotic boundary condition. The Laplace transforms of $\frac{\partial q_{l}}{\partial x}$ and $q_{l}$ are related by

$$
\frac{\partial \hat{q}_{l}}{\partial x}(x, y ; s)=\lambda_{l}(s) \hat{q}_{l}(x, y ; s) .
$$

The steepest descent result involves the restriction of the transforms to a neighborhood of $s^{*}\left(\tilde{\gamma}_{l}\right)$. An asymptotic expansion of the $x$-derivative may then be obtained by replacing $\lambda_{l}(s)$ by its Taylor series about the critical value of $s$ :

$$
\lambda_{l}(s) \approx \lambda_{l}\left(s^{*}\left(\tilde{\gamma}_{l}\right)\right)+\lambda_{l}^{1}\left(s-s^{*}\left(\tilde{\gamma}_{l}\right)\right)+\lambda_{l}^{2}\left(s-s^{*}\left(\tilde{\gamma}_{l}\right)\right)^{2}+\cdots .
$$

Using, for example, the first two terms, we have

$$
\frac{\partial q_{l}}{\partial x} \sim\left(\lambda_{l}^{0}+\lambda_{l}^{1} \frac{\partial}{\partial t}\right) q_{l}
$$

These may be substituted into $\left(j_{i}\right)$ to finally obtain a condition on $u_{l}$. The time-derivative is brought outside the integral to further simplify the expression. This involves the neglect of terms from the limits of integration, which should 
be exponentially small. The asymptotic boundary condition we propose is, then, given by

$$
\frac{\partial u_{l}}{\partial x}=\left(\lambda_{l}^{0}+\lambda_{l}^{1} \frac{\partial}{\partial t}\right) u_{l}
$$

A hierarchy of conditions may be obtained by use of more terms in the Taylor series. These would involve derivatives of higher order. Their stable implementation would require the use of Padé approximants, as discussed by Engquist and Majda [5]. For example, a potential approximation at the next order is

$$
\left(1-\frac{\lambda_{l}^{2}}{\lambda_{l}^{1}}\left(\frac{\partial}{\partial t}-s^{*}\right)\right)\left(\frac{\partial}{\partial x}-\lambda_{l}\left(s^{*}\right)\right) u_{l}=\lambda_{l}^{1}\left(\frac{\partial}{\partial t}-s^{*}\right) u_{l} .
$$

The condition derived involves only one normal mode, $u_{l}$. We may, however, apply it directly to $u$ if $l$ is such that $\mathfrak{R}\left(\lambda_{l}^{0}\right)$ is maximized. For problems where a small number of modes have similar minimum decay rates, a product boundary condition is used. Also, if the critical $s^{*}$ is imaginary, we must include its complex conjugate in the product. We have in general

$$
\left[\prod_{l_{i}}\left(\frac{\partial}{\partial x}-\lambda_{l_{i}}^{0}-\lambda_{l_{i}}^{1} \frac{\partial}{\partial t}\right) \text { (complex conjugate) }\right] u=0 .
$$

We have never used more than two modes.

\section{NUMERICAL CONSIDERATIONS}

In order to apply the asymptotic boundary conditions derived above, we must find $\tilde{\gamma}_{l}$ as well as the first two terms of the Taylor series of $\lambda_{l}$. For fixed $s$, the substitution $w=\lambda v$ in conjunction with an appropriate discretization of the $y$-derivatives leads to a generalized matrix eigenvalue problem:

$$
M(s) r=\lambda L r, \quad r=\left(\begin{array}{c}
\tilde{v} \\
\tilde{w}
\end{array}\right) .
$$

This may be solved using standard linear algebra software which implements, say, the QZ algorithm [6]. An analysis of the discretization error is given by Kreiss [17]. The asymptotic expansion requires that $s$ be imaginary and $\frac{d \lambda}{d s}$ be real. As $s$ varies along the imaginary axis, the latter implies a maximum or minimum for $\mathfrak{R}(\lambda)$. Our problem is, then, to maximize $\mathfrak{R}\left(\lambda_{l}(s)\right)$ as $s$ varies over the imaginaries. This is a line search problem for which many strategies have been developed, though they may be expensive to carry out. (See, e.g., Dennis and Schnabel [3].) For many of our examples, $s=0$ has been the solution. A necessary condition for this is that $\frac{d \lambda}{d s}(0)$ be real and negative. This may be checked by solving (44) only once.

Once the critical value of $s$ has been found, we must compute $\lambda_{l}^{1}$. Differentiating (44) with respect to $s$ yields

$$
(M-\lambda L) \frac{d r}{d s}=\left(\frac{d \lambda}{d s} L-\frac{d M}{d s}\right) r .
$$


Since the matrix on the left is singular, $\lambda_{l}^{1}=\frac{d \lambda_{l}}{d s}$ may be obtained from a compatibility condition. If $\tilde{r}$ is a left null vector we have

$$
\lambda_{l}^{1}=\frac{\tilde{r}^{T} \frac{d M}{d s} r}{\tilde{r}^{T} L r} .
$$

The cost of this computation is typically negligible in comparison with the cost of finding the critical frequency. Expressions for more terms in the Taylor series can be similarly found.

There are a variety of reasonable numerical implementations of the boundary conditions. For conditions involving derivatives of at most first order, many stable discretizations are known. Product boundary conditions (for wave equations) are studied by Higdon [16]. He develops the useful principle of employing products of stable discretizations. We have successfully employed this procedure throughout our numerical experiments.

\section{ERROR ESTIMATES}

Estimating the error caused by the introduction of an artificial boundary can be broken up into two parts. The first is to estimate the residual resulting from the application of the boundary condition to the exact solution. The complete error estimate then follows from estimates of the solution of the initial-boundary value problem in terms of inhomogeneous boundary data. The latter is simply a proof of well-posedness.

For reference we write down the problem satisfied by the error, $e(x, y, t)$.

\section{Problem 1.}

$$
\begin{gathered}
\text { Le }=0, \quad(x, y) \in(0, \tau) \times\left(y_{0}, y_{1}\right), \\
e(x, y, 0)=0, \\
D_{j} e\left(x, y_{j}, t\right)=0, \quad j=0,1, \\
E_{0} e(0, y, t)=0, \\
B e(\tau, y, t)=B u(\tau, y, t) .
\end{gathered}
$$

Here, $L$ is the differential operator appearing in (4) and $B$ is the asymptotic boundary operator appearing in (43).

A general approach to the investigation of the effect of the boundary conditions on the well-posedness of an initial-boundary value problem is to freeze coefficients at each point of the boundary and to study solutions of the frozencoefficient system. In particular, we must show that no solutions of the frozencoefficient problem of the form

$$
e^{\kappa t+\mu x+i \alpha y} \nu, \quad \mathfrak{R} \kappa \geq 0, \mathfrak{R} \mu>0,
$$

are in the null space of the boundary operator. For problems with decay in the dominant wave groups, that is $\Re \lambda_{l_{i}}^{0}<0$ in (43), it is clear that an eigensolution 
satisfying the conditions above cannot exist, as

$$
\mathfrak{R}\left(\mu-\lambda_{l_{i}}^{0}-\lambda_{l_{i}}^{1} \kappa\right)>0
$$

(Recall that $\lambda_{l_{i}}^{1}$ is real and negative.)

Translating this condition into a bonafide proof of well-posedness requires further assumptions on the coefficients of (4). Indeed, the conditions we have constructed may result in an overdetermined problem if $A$ is rank-deficient. Complete discussions are given by Eidel'man [4] for the parabolic case and by Strikwerda [19] for incompletely parabolic systems, though in general their results require boundary operators of low order. See also the more stringent requirement of dissipativity introduced by Barry, Bielak, and MacCamy [1], which may be necessary for the estimates used below. We will simply assume that Problem 1 is well-posed. We introduce appropriate Sobolev norms in the interior, $\|\cdot\|_{(0, \tau) \times\left(y_{0}, y_{1}\right)}$, and on the boundary, $\|\cdot\|_{\left(y_{0}, y_{1}\right)}$, and define

$$
\begin{gathered}
\|w\|_{1}=\int_{0}^{\infty}\|w(\cdot, \cdot, t)\|_{(0, \tau) \times\left(y_{0}, y_{1}\right)} d t \\
\|w\|_{1, x}=\int_{0}^{\infty}\|w(x, \cdot, t)\|_{\left(y_{0}, y_{1}\right)} d t
\end{gathered}
$$

and, for functions of $t$,

$$
|w|_{1}=\int_{0}^{\infty}|w(t)| d t
$$

We then make:

Assumption 1. There exists $C>0$ independent of $\tau$ such that

$$
\|e\|_{1} \leq C\|B u\|_{1, \tau} \text {. }
$$

(In what follows we mean all constants to be independent of $\tau$ unless otherwise stated.)

The requirement that $C$ be independent of $\tau$ can easily be dropped, though we must be able to estimate its growth in order to estimate the rate of convergence of the solution on the truncated domain. For example, in the error analysis of asymptotic boundary conditions for second-order scalar hyperbolic equations in exterior domains, as given by Bayliss and Turkel [2] and Hariharan and Hagstrom [14], algebraic growth of $C$ with $\tau$ is encountered. For the problems under consideration, however, Assumption 1 is typically satisfied owing to the exponential decay of solutions. An interesting possibility is to use weighted norms in $x$, in which case $C$ may decrease with increasing $\tau$.

We proceed to estimate $\|B u\|_{1, \tau}$. We make a variety of simplifying assumptions, as our main purpose is to extract the dependence of the error on $\tau$ as well as to give the flavor of the necessary computations. Many of the assumptions could be relaxed somewhat, though their verification for any practical problem 
is likely to be difficult. We freely quote error estimates from the asymptotic theory of integrals with large parameters. These may be found in any of a variety of texts on the subject, for example Sirovich [18].

We make, then, the following assumptions:

Assumption 2. For all $l \in \mathscr{N}$ and all $\gamma \in[0, \infty)$ there exists a unique (up to complex conjugation) $s^{*}(\gamma)$ satisfying (19), and the contour of integration in (17) can be deformed to the steepest descent path through $s^{*}$ without passing through singularities of the integrand. Furthermore, for $\tau$ sufficiently large, $D^{j}\left(q_{l}-\phi_{l}\right)$ is absolutely integrable in $\gamma$ and the integral is uniformly bounded in $l$, where $D$ represents a derivative in $t$ or $x$ and $j \leq 2$.

Assumption 3. There exists $l_{0} \in \mathcal{N}$ such that a unique $\tilde{\gamma}_{l_{0}}$ exists at which $\mathfrak{R}\left(\gamma s^{*}+\lambda_{l_{0}}\left(s^{*}\right)\right) \equiv \mu_{0}$ attains a global maximum. If $s^{*}\left(\tilde{\gamma}_{l_{0}}\right)=0$, then, for $l=l_{0}, \mathfrak{I}\left(s^{*}(\gamma)\right)=0$ for all $\gamma$ in a neighborhood of $\tilde{\gamma}_{l_{0}}$. Furthermore, there exists $\delta>0$ such that $\mathfrak{R}\left(\gamma s^{*}+\lambda_{l}\left(s^{*}\right)\right) \leq \mu_{0}-\delta$ for all $\gamma$ and $l \neq l_{0}$.

Assumption 4. There exists $z$ such that, for $0 \leq j \leq 2$,

$$
\sum_{l \neq l_{0}} \int_{0}^{\infty} e^{z \mu_{l}(p)} h_{l, j}(p) d p \leq K_{0}<\infty,
$$

where

$$
\begin{gathered}
\mu_{l}(p)=\mathfrak{R}\left(s^{*}(p) p+\lambda_{l}\left(s^{*}(p)\right)\right), \\
h_{l, j}(p)=\frac{\left\|v_{l}\left(\cdot, s^{*}(p)\right)\right\|_{\left(y_{0}, y_{1}\right)}}{\sqrt{\left|\lambda_{l}^{\prime \prime}\left(s^{*}\right)\right|}}\left(\left|s^{*}(p)\right|^{j}+\left|\lambda_{l}\left(s^{*}(p)\right)\right|^{j}\right) .
\end{gathered}
$$

(We will always take $\tau>z$.)

Assumption 5. There exist $K_{1}>0, K_{2}>0$, and $K_{3}>0$ such that

$$
\begin{gathered}
\left|c_{l}\right|_{1} \leq K_{1}\left|c_{l_{0}}\right|_{1}, \quad l \neq l_{0}, \\
\left\|u_{l_{0}}\right\|_{1, \tau} \geq K_{2}\left|c_{l_{0}}\right|_{1}\left\|q_{l_{0}}\right\|_{1, \tau}, \\
\left|c_{l_{0}}\right|_{1} \leq K_{3}\|u\|_{1,0} .
\end{gathered}
$$

Assumptions 2-4 are constraints on the coefficients of equation (4). They are easily verified in the interesting special case of the advection diffusion equation with constant coefficients and the usual (e.g., Dirichlet or Neumann) conditions on the channel wall. Assumption 5 is in effect a restriction to signal data which excites the dominant wave group. If the data is such that only a certain wave group is excited, that information should be used to modify the asymptotic analysis.

The main estimates are derived in the following collection of lemmas, whose proofs depend on the validity of Assumptions 2-5. 
Lemma 1. There holds, as $\tau \rightarrow \infty$,

$$
\left\|B q_{l_{0}}\right\|_{1, \tau}=O(1 / \tau) \cdot\left\|q_{l_{0}}\right\|_{1, \tau} .
$$

Proof. We have, by our assumptions on the properties of the transforms,

$$
\left\|q_{l_{0}}\right\|_{1, \tau}=\left\|\phi_{l_{0}}\right\|_{1, \tau}(1+O(1 / \tau)) \text {. }
$$

Writing out the integral expression for $\left\|\phi_{l_{0}}\right\|_{1, \tau}$ and introducing the change of variables $p=t / \tau$ yields

$$
\sqrt{\frac{\tau}{2 \pi}} \int_{0}^{\infty} e^{\tau f(p)}|\cos (\tau g(p)+\theta(p))| h(p) d p
$$

Here,

$$
\begin{gathered}
f(p)=\mathfrak{R}\left(s^{*}(p) p+\lambda_{l_{0}}\left(s^{*}(p)\right)\right), \\
g(p)=\mathfrak{I}\left(s^{*}(p) p+\lambda_{l_{0}}\left(s^{*}(p)\right)\right), \\
h(p)=\frac{\left\|v_{l_{0}}\left(\cdot ; s^{*}(p)\right)\right\|_{\left(y_{0}, y_{1}\right)}}{\sqrt{\left|\lambda_{l_{0}}^{\prime \prime}\left(s^{*}(p)\right)\right|}} \\
\theta(p)=-\frac{1}{2} \arg \left(\lambda_{l_{0}}^{\prime \prime}\left(s^{*}(p)\right)\right) .
\end{gathered}
$$

Again, by assumption, $f(p)$ has a maximum at $p=\tilde{\gamma}_{l_{0}} \equiv p_{0}$. The approximation of this integral by Laplace's method is slightly complicated by the presence of the absolute value of the oscillatory term. There are two cases to consider. If $s^{*}\left(p_{0}\right)=0$, then, by Assumption 3, it is 0 in a neighborhood of $p_{0}$, and by direct computation we find that $g(p)$ is constant. Then Laplace's formula may be directly applied. If $s^{*}\left(p_{0}\right) \neq 0$, then $g^{\prime}\left(p_{0}\right)=\mathfrak{I}\left(s^{*}\right) \neq 0$. Then the generalization of Laplace's formula given in Theorem 2 of the appendix applies. In each case the result is

$$
\left\|\phi_{l_{0}}\right\|_{1, \tau}=K e^{\tau \mu_{0}}\left(1+o\left(1 / \tau^{1 / 2}\right)\right),
$$

where $K$ is independent of $\tau$. Similarly, asymptotic expansions of $B\left(\frac{\partial}{\partial t}, \frac{\partial}{\partial x}\right) q_{l_{0}}$ can be computed. In particular, we have

$$
\left\|B q_{l_{0}}\right\|_{1, \tau}=\left\|B \phi_{l_{0}}\right\|_{1, \tau}(1+O(1 / \tau)) .
$$

As above, we consider the integral expression for $\left\|B \phi_{l_{0}}\right\|$ :

$$
\sqrt{\frac{\tau}{2 \pi}} \int_{0}^{\infty} e^{\tau f(p)}|\cos (\tau g(p)+\tilde{\theta}(p))| \tilde{h}(p) d p .
$$

Here, $f$ and $g$ are as above, while $\tilde{\theta}$ and $\tilde{h}$ are determined by

$$
\begin{aligned}
& \tilde{h}(p)=\left\|v_{l_{0}}\left(\cdot ; s^{*}(p)\right)\right\|_{\left(y_{0}, y_{1}\right)} \frac{\left|B\left(s^{*}(p), \lambda_{l_{0}}\left(s^{*}(p)\right)\right)\right|}{\sqrt{\left|\lambda_{l_{0}}^{\prime \prime}\left(s^{*}(p)\right)\right|}}, \\
& \tilde{\theta}(p)=\arg \left(B\left(s^{*}(p), \lambda_{l_{0}}\left(s^{*}(p)\right)\right)\right)-\frac{1}{2} \arg \left(\lambda_{l_{0}}^{\prime \prime}\left(s^{*}(p)\right)\right) .
\end{aligned}
$$


Again, we will compute an asymptotic approximation to this integral using Laplace's formula if $s^{*}\left(p_{0}\right)=0$, or Theorem 2 if it is not. We note that $B$ has been chosen to have a simple zero at $p=p_{0}$, which implies that

$$
\tilde{h}(p)=O\left(p-p_{0}\right), \quad p \rightarrow p_{0} .
$$

We thereby conclude

$$
\left\|B \phi_{l_{0}}\right\|_{1, \tau}=O(1 / \tau)\left\|\phi_{l_{0}}\right\|_{1, \tau} .
$$

The conclusion of the lemma follows directly.

Lemma 2. We have, as $\tau \rightarrow \infty$,

$$
\left\|B u_{l_{0}}\right\|_{1, \tau}=O(1 / \tau)\left\|u_{l_{0}}\right\|_{1, \tau} .
$$

Proof. By direct computation,

$$
B u_{l_{0}}=c_{l_{0}} * B q_{l_{0}}+\text { boundary terms. }
$$

The asymptotic analysis indicates that the boundary terms are exponentially small. We then have, using Lemma 1 and Assumption 5,

$$
\begin{aligned}
\left\|B u_{l_{0}}\right\|_{1, \tau} & \approx\left\|c_{l_{0}} * B q_{l_{0}}\right\|_{1, \tau} \leq\left|c_{l_{0}}\right|_{1}\left\|B q_{l_{0}}\right\|_{1, \tau} \\
& \leq O(1 / \tau)\left|c_{l_{0}}\right|_{1}\left\|q_{l_{0}}\right\|_{1, \tau} \leq O(1 / \tau)\left\|u_{l_{0}}\right\|_{1, \tau} .
\end{aligned}
$$

Lemma 3. There exists $\eta>0$ such that

$$
\left\|B\left(u-u_{l_{0}}\right)\right\|_{1, \tau}=O\left(e^{-\eta \tau}\right)\left\|u_{l_{0}}\right\|_{1, \tau} .
$$

Proof. We directly estimate $\left\|B\left(u-u_{l_{0}}\right)\right\|$ :

$$
\left\|B\left(u-u_{l_{0}}\right)\right\|_{1, \tau} \approx\left\|\sum_{l \neq l_{0}} c_{l} * B q_{l}\right\|_{1, \tau} \leq K\left|c_{l_{0}}\right|_{1} \sum_{l \neq l_{0}}\left\|B \phi_{l}\right\|_{1, \tau},
$$

where we have used Assumptions 2 and 5. From the integral representation of $\left\|\phi_{l}\right\|$ we have

$$
\sum_{l \neq l_{0}}\left\|B \phi_{l}\right\|_{1, \tau} \leq \sqrt{\frac{\tau}{2 \pi}} \sum_{l \neq l_{0}} \int_{0}^{\infty} e^{\tau f_{l}(p)} \tilde{h}_{l}(p) d p
$$

where

$$
\begin{gathered}
f_{l}(p)=\mathfrak{R}\left(s^{*}(p) p+\lambda_{l}\left(s^{*}(p)\right)\right), \\
\tilde{h}_{l}(p)=\left\|v_{l}\left(\cdot ; s^{*}(p)\right)\right\|_{\left(y_{0}, y_{1}\right)} \frac{\left|B\left(s^{*}(p), \lambda_{l}\left(s^{*}(p)\right)\right)\right|}{\sqrt{\left|\lambda_{l}^{\prime \prime}\left(s^{*}(p)\right)\right|}} .
\end{gathered}
$$

However, by Assumptions 4 and 3, the expression on the right is bounded by

$$
K_{0} \sqrt{\frac{\tau}{2 \pi}} e^{(\tau-z)\left(\mu_{0}-\delta\right)}
$$


We have, therefore, for some constant $K_{4}$,

$$
\left\|B\left(u-u_{l_{0}}\right)\right\|_{1, \tau} \leq \frac{K_{4}}{\sqrt{\tau}} e^{-\delta \tau}\left(e^{\tau \mu_{0}}\left|c_{l_{0}}\right|_{1}\right) .
$$

By Assumption 5 and the asymptotic expansion of $\left\|q_{l_{0}}\right\|$ computed in the proof of Lemma 1 , the term in parentheses is bounded by $\left\|u_{l_{0}}\right\|_{1, \tau}$. We have thus shown that $\eta>0$ can be chosen such that

$$
\left\|B\left(u-u_{l_{0}}\right)\right\|=O\left(e^{-\eta \tau}\right)\left\|u_{l_{0}}\right\|_{1, \tau}
$$

completing the proof of the lemma.

Lemma 4. There exists $K$ such that

$$
\|B u\|_{1, \tau} \leq K \frac{e^{\tau \mu_{0}}}{\tau}\|u\|_{1,0}
$$

Proof. We have, by Lemmas 2 and 3,

$$
\|B u\|_{1, \tau} \leq\left\|B u_{l_{0}}\right\|_{1, \tau}+\left\|B\left(u-u_{l_{0}}\right)\right\|_{1, \tau} \leq O(1 / \tau)\left\|u_{l_{0}}\right\|_{1, \tau}
$$

Furthermore, using the asymptotic expansion of $\left\|\phi_{l_{0}}\right\|_{1, \tau}$ computed in the proof of Lemma 1, along with Assumption 5, we find

$$
\left\|u_{l_{0}}\right\|_{1, \tau} \leq K_{5}\left|c_{l_{0}}\right|_{1}\left\|\phi_{l_{0}}\right\|_{1, \tau} \leq K_{6} e^{\tau \mu_{0}}\|u\|_{1,0} .
$$

Combining these equations yields the statement of the lemma.

Finally, by combining Assumption 1 with Lemma 4 we obtain the desired error estimate:

Theorem 1. Suppose $e$ is the solution of Problem 1, and Assumptions 1-5 hold. Then for $\tau$ sufficiently large there exists $\bar{K}$ independent of $\tau$ and the data such that

$$
\|e\|_{1} \leq \bar{K} \frac{e^{\tau \mu_{0}}}{\tau}\|u\|_{1,0}
$$

\section{CONCLUDING REMARKS}

We have developed a general technique for the construction of asymptotic boundary conditions for problems with dissipative wave propagation. Theorem 1 clearly implies the convergence of the solution on the truncated domain as $\tau \rightarrow \infty$, if $\mu_{0} \leq 0$. The factor of $1 / \tau$, which is a direct consequence of the use of our asymptotic boundary conditions, is of importance when $\mu_{0}$ is small. Precisely such a situation occurs when our technique is applied to the incompressible Navier-Stokes equations for moderate to large Reynolds numbers. This is studied in detail in [10]. Applications to other problems in computational mechanics are also under consideration. 


\section{APPENDIX. ASYMPTOTIC EXPANSION OF AN INTEGRAL}

In this section we compute the asymptotic expansion as $\tau \rightarrow \infty$ of the following integral, which is needed for the derivation of our error estimates:

$$
I=\int_{p_{-}}^{p_{+}} e^{\tau f(p)}|\cos (\tau g(p)+\theta(p))| h(p) d p .
$$

The assumptions we will make are:

Assumption 6. (a) The function $f$ attains a global maximum at $p_{0} \in\left(p_{-}, p_{+}\right)$ and is thrice continuously differentiable in a neighborhood of $p_{0}$ with $f^{\prime \prime}\left(p_{0}\right)$ $\neq 0$.

(b) The function $g$ is twice continuously differentiable in a neighborhood of $p_{0}$ and $g^{\prime}\left(p_{0}\right) \neq 0$.

(c) The function $h$ is bounded outside a neighborhood of $p_{0}$, and near $p=p_{0}$ satisfies

$$
h(p)=h_{0}\left(p-p_{0}\right)^{m}+h_{1}\left(p-p_{0}\right)^{m+1}\left(1+O\left(p-p_{0}\right)\right),
$$

where $h_{0} \neq 0$ and, if $m$ is odd,

$$
h_{1}-(m+2) h_{0} \frac{f^{\prime \prime \prime}\left(p_{0}\right)}{3 f^{\prime \prime}\left(p_{0}\right)} \neq 0 .
$$

(d) The function $\theta$ is continuous in a neighborhood of $p_{0}$.

(e) If $p_{ \pm}= \pm \infty$, then $f(p) \rightarrow-\infty$ at least algebraically as $|p| \rightarrow \infty$.

The smoothness and decay conditions can be relaxed somewhat. If $m$ is odd and (95) does not hold, we must simply consider higher-order terms in the expansion of $f$ and $h$.

Despite its apparent simplicity, this integral does not seem to be discussed in the standard references. Because of the presence of the oscillatory term, Laplace's method cannot be directly applied. Intuitively, we expect to obtain the dominant behavior by replacing $|\cos (\tau g+\theta)|$ by its mean value. This is established in the following theorem. Although we have been able to estimate the contribution of the oscillatory terms, we have been unable to compute it to leading order for general $f$ and $g$.

Theorem 2. If Assumption 6 holds, then as $\tau \rightarrow \infty$,

$$
I \sim \frac{2 K_{m}}{\pi} e^{\tau f\left(p_{0}\right)}\left(\frac{-\tau f^{\prime \prime}\left(p_{0}\right)}{2}\right)^{-\gamma_{m}} \Gamma\left(\gamma_{m}\right),
$$

where

$$
\begin{gathered}
\gamma_{m}= \begin{cases}(m+1) / 2, & m \text { even }, \\
(m+2) / 2, & m \text { odd },\end{cases} \\
K_{m}= \begin{cases}h_{0}, & m \text { even }, \\
h_{1}-(m+2) h_{0} f^{\prime \prime \prime}\left(p_{0}\right) / 3 f^{\prime \prime}\left(p_{0}\right), & m \text { odd } .\end{cases}
\end{gathered}
$$


Proof. We begin by replacing $|\cos (\tau g+\theta)|$ by its Fourier series:

$$
\begin{gathered}
|\cos \theta|=\frac{2}{\pi}+\sum_{n=1}^{\infty} c_{n} \cos 2 n \theta, \\
c_{n}=\frac{4}{\pi} \frac{(-1)^{n+1}}{4 n^{2}-1} .
\end{gathered}
$$

As the series converges uniformly, $I$ may be expressed as the sum of integrals:

$$
I=\int_{p_{-}}^{p_{+}} \frac{2}{\pi} e^{\tau f(p)} h(p) d p+\sum_{n=1}^{\infty} c_{n} I_{n}
$$

where

$$
I_{n}=\int_{p_{-}}^{p_{+}} e^{\tau f(p)} \cos (2 n(\tau g(p)+\theta(p))) h(p) d p .
$$

The asymptotic expansion of the first integral follows directly from the use of Laplace's method and yields the results stated in the theorem [18]. What remains is to show that the contribution of the oscillatory integrals is of lower order. Therefore, we consider the asymptotic analysis of $I_{n}$.

In the usual way we restrict the integral to a small, fixed interval about $p_{0}$, introducing an error which is exponentially small. Using the fact that $g^{\prime}\left(p_{0}\right) \neq$ 0 , we are able to make a change of variables so that the remaining integral becomes

$$
e^{\tau f\left(p_{0}\right)} \int_{|u|<\varepsilon} e^{-\tau F(u)} \cos (n \tau u) \widetilde{H}(u, \tau) d u
$$

where

$$
F(u)=F_{2} u^{2}+F_{3} u^{3}+o\left(u^{3}\right), \quad F_{2}>0
$$

and

$$
\begin{gathered}
\tilde{H}(u, \tau)=H(u)(1+O(1 / \tau)), \\
H(u)=H_{0} u^{m}+H_{1} u^{m+1}+o\left(u^{m+1}\right), \quad H_{0} \neq 0 .
\end{gathered}
$$

We break this integral into two parts,

$$
\begin{gathered}
I_{n}=e^{\tau f\left(p_{0}\right)}\left(I_{n}^{1}+I_{n}^{2}\right), \\
I_{n}^{1}=\int e^{-\tau F_{2} u^{2}} \cos (n \tau u) H_{0} u^{m} d u, \\
I_{n}^{2}=\int e^{-\tau F_{2} u^{2}} \cos (n \tau u) \Delta(u, \tau) d u .
\end{gathered}
$$

Here we have

$$
\Delta(u, \tau)=\left(e^{-\tau\left(F(u)-F_{2} u^{2}\right)}-1\right) \widetilde{H}(u, \tau)+\left(\widetilde{H}(u, \tau)-H_{0} u^{m}\right) .
$$


The asymptotic analysis of $I_{n}^{1}$ may be carried out using the method of steepest descent. The exponent has a single critical point at $u=i n / 2 F_{2}$, leading to an exponentially small contribution. To estimate $I_{n}^{2}$, we first rewrite $\Delta$ :

$$
\Delta(u, \tau)=\tau u^{m+3} \delta_{1}(u, \tau)+u^{m+1} \delta_{2}(u, \tau),
$$

where $\delta_{1}$ and $\delta_{2}$ are bounded functions. Introducing the change of variables

$$
v=\sqrt{\tau F_{2}} u
$$

we obtain

$$
\begin{aligned}
I_{n}^{2}=\tau^{-(m+2) / 2} \int_{v_{-}}^{v_{+}} & e^{-v^{2}} \cos \left(\sqrt{\frac{\tau}{F_{2}}} n v\right) \\
& \cdot\left(v^{m+3} d_{1}(v, \tau)+v^{m+1} d_{2}(v, \tau)\right) d v .
\end{aligned}
$$

Here, $d_{1}$ and $d_{2}$ are uniformly bounded and $v_{ \pm}= \pm \sqrt{\tau F_{2}} \varepsilon$. For convenience we extend the domain of integration to the entire real line, extending $d_{1}$ and $d_{2}$ so that they remain uniformly bounded. As the integrand is absolutely integrable on the entire real line, uniformly in $\tau$, the additional term is $o\left(\tau^{-(m+2) / 2}\right)$. To estimate the remaining integral, we essentially use the Riemann-Lebesgue lemma, modified to take account of the dependence of the nonoscillatory terms on $\tau$. We have

$$
I_{n}^{2}=\tau^{-(m+2) / 2} I_{n}^{3}+o\left(\tau^{-(m+2) / 2}\right)
$$

where, following the usual transformation,

$$
I_{n}^{3}=\frac{1}{2} \int_{-\infty}^{+\infty}\left(G(v, \tau)-G\left(v+\sqrt{\frac{F_{2}}{n^{2} \tau}}, \tau\right)\right) \cos \left(\sqrt{\frac{\tau}{F_{2}}} n v\right) d v
$$

Here, $G$ is given by

$$
G(v, \tau)=e^{-v^{2}}\left(v^{m+3} d_{1}(v, \tau)+v^{m+1} d_{2}(v, \tau)\right) .
$$

Again, $G$ is absolutely integrable, uniformly in $\tau$, so we need only show that

$$
\lim _{\tau \rightarrow \infty}\left(G(v, \tau)-G\left(v+\sqrt{\frac{F_{2}}{n^{2} \tau}}, \tau\right)\right)=0 .
$$

Making use of the fact that for fixed $v$ the limit $\tau \rightarrow \infty$ implies $u \rightarrow 0$, we have

$$
\lim _{\tau \rightarrow \infty} d_{1}(v, \tau)=\lim _{u \rightarrow 0} \frac{F_{2}}{v^{2}} \frac{\left(e^{-v^{2}\left(F(u) / F_{2} u^{2}-1\right)}-1\right)}{u} \frac{\widetilde{H}(u, \tau)}{u^{m}}=-F_{3} H_{0}
$$

and

$$
\lim _{\tau \rightarrow \infty} d_{2}(v, \tau)=\lim _{u \rightarrow 0} \frac{\left(H(u)\left(1+O\left(u^{2}\right)\right)-H_{0} u^{m}\right)}{u^{m+1}}=H_{1} .
$$


From these we may conclude that (117) holds and, therefore, that $\lim _{\tau \rightarrow \infty} I_{n}^{3}=$ 0 . We have shown, then, that $I_{n}^{2}=o\left(\tau^{-(m+2) / 2}\right)$. This, in turn, implies

$$
I_{n}=o\left(e^{\tau f\left(p_{0}\right)} \tau^{-(m+2) / 2}\right), \quad \tau \rightarrow \infty .
$$

As the bounds obtained above may be made independent of $n$, we conclude that the contribution of the oscillatory terms is dominated by the contribution of the first term in the Fourier series, completing the proof of the theorem.

It should be noted that we have not computed the leading order asymptotics of $I_{n}$. One might at first glance expect that $I_{n}^{1}$ determines the leading order behavior. We have, however, shown that it is exponentially small, while the bounds obtained for the remaining terms decay algebraically.

\section{BIBLIOGRAPHY}

1. A. Barry, J. Bielak, and R. C. MacCamy, On absorbing boundary conditions for wave propagation, J. Comp. Phys. 79 (1988), 449-468.

2. A. Bayliss and E. Turkel, Radiation boundary conditions for wave-like equations, Comm. Pure Appl. Math. 33 (1980), 707-725.

3. J. E. Dennis, Jr. and R. B. Schnabel, Numerical methods for unconstrained optimization and nonlinear equations, Prentice-Hall, Englewood Cliffs, N.J., 1983.

4. S. D. Eidel' man, Parabolic systems, North-Holland, Amsterdam, 1969.

5. B. Engquist and A. Majda, Absorbing boundary conditions for the numerical simulation of waves, Math. Comp. 31 (1977), 629-651.

6. G. H. Golub and C. F. van Loan, Matrix computations, 2nd ed., Johns Hopkins Univ. Press, Baltimore, 1989.

7. B. Gustafsson and H.-O. Kreiss, Boundary conditions for time dependent problems with an artificial boundary, J. Comput. Phys. 30 (1979), 333-351.

8. T. Hagstrom, Boundary conditions at outflow for a problem with transport and diffusion, J. Comput. Phys. 69 (1987), 69-80.

9. __ Asymptotic expansions and boundary conditions for time-dependent problems, SIAM J. Numer. Anal. 23 (1986), 948-958.

10. Conditions at the downstream boundary for simulations of viscous, incompressible flow, SIAM J. Sci. Statist. Comput. (submitted).

11. T. Hagstrom and H. B. Keller, Numerical calculation of traveling wave solutions of nonlinear parabolic equations, SIAM J. Sci. Statist. Comput. 7 (1986), 978-988.

12. L. Halpern, Artificial boundary conditions for the linear advection diffusion equation, Math. Comp. 46 (1986), 425-438.

13. L. Halpern and M. Schatzman, Artificial boundary conditions for viscous incompressible flows, SIAM J. Math. Anal. 20 (1989), 308-353.

14. S. I. Hariharan and T. Hagstrom, Far field expansion for anisotropic waves, Computational Acoustics, vol. 2 (D. Lee, A. Cakmak, and R. Vichnevetsky, eds.), North-Holland, Amsterdam, 1990, pp. 283-294.

15. R. L. Higdon, Initial-boundary value problems for linear hyperbolic systems, SIAM Rev. 28 (1986), 177-217.

16. $\ldots$, Numerical absorbing boundary conditions for the wave equation, Math. Comp. 49 (1987), 65-90.

17. H.-O. Kreiss, Difference approximations for boundary and eigenvalue problems for ordinary differential equations, Math. Comp. 26 (1972), 605-624. 
18. L. Sirovich, Techniques of asymptotic analysis, Appl. Math. Sci., Vol. 2, Springer-Verlag, New York, 1971.

19. J. C. Strikwerda, Initial boundary value problems for incompletely parabolic systems, Comm. Pure Appl. Math. 30 (1977), 797-822.

Department of Applied Mathematics and Statistics, SUNY at Stony Brook, Stony BROOK, NEW YORK 11794

Current address: Department of Mathematics and Statistics, The University of New Mexico, Albuquerque, NM 87131

E-mail address: hagstrom@euler.unm.edu 